\title{
A high-sensitivity large volume cryogenic detector for radon in gas
}

\author{
M. Wojcik, ${ }^{1 *}$ G. Zuzel ${ }^{2}$ \\ ${ }^{1}$ M. Smoluchowski Institute of Physics, Reymonta 4, 30-059 Cracow, Poland \\ ${ }^{2}$ Max Planck Institute for Nuclear Physics, Saupfercheckweg 1, 69117 Heidelberg, Germany
}

(Received March 29, 2007)

\begin{abstract}
A new detector for ${ }^{222} \mathrm{Rn}$ measurements in gases is described. It is based on the low temperature collection of radon on a stainless steel plate and subsequent counting of emitted alpha-particles using an ion-implanted silicon detector. A very low background of 25 counts/day for the ${ }^{222} \mathrm{Rn}$ energy window was achieved. High detection efficiency of $32 \%$ ( $2 \pi$ geometry) and the large 65 liter volume of the detector allow radon measurements at the level of $12 \mathrm{mBq} / \mathrm{m}^{3}$ with $30 \%$ accuracy (relative error). Behavior of the alpha-emitting radon daughters born on the cooled stainless steel plate, ${ }^{218} \mathrm{Po}$ and ${ }^{214} \mathrm{Po}$, was also analyzed.
\end{abstract}

\section{Introduction}

Currently the most sensitive technique for ${ }^{222} \mathrm{Rn}$ detection is based on the counting using ultra low-level proportional counters, ${ }^{1}$ originally built for the GALLEX/GNO experiment. ${ }^{2}$ However, use of these detectors requires pre-concentration of ${ }^{222} \mathrm{Rn}$, followed by purification of the gas sample, and a complicated detector filling procedure. For a single measurement it is possible to reach the absolute detection limit at the level of $30 \mu \mathrm{Bq}$.

Another approach is to use electrostatic chambers, ${ }^{3,4}$ where the positively charged ${ }^{222} \mathrm{Rn}$ daughters are collected on the active surface of the semiconductor diode where their alpha-decays are registered. To reach a high and stable collection efficiency the semiconductor detector must be placed on relatively high voltage (several kV). The absolute sensitivity of such detectors lies at the level of some $\mathrm{mBq}$; combined with large active volumes this allows measurements of ${ }^{222} \mathrm{Rn}$ concentrations in gases slightly below $1 \mathrm{mBq} / \mathrm{m}^{3}$.

Lucas cell scintillation chambers are also very convenient, ${ }^{5}$ especially for ${ }^{222} \mathrm{Rn}$ monitoring in underground laboratories or clean rooms. However, they are usually not sensitive enough to detect ${ }^{22} \mathrm{Rn}$ at very low levels, mainly because of rather high background count rate due to scintillator impurities. Achievable sensitivity reaches $0.5 \mathrm{~Bq} / \mathrm{m}^{3}$.

In this paper we describe a prototype of a new cryogenic detector for ${ }^{222} \mathrm{Rn}$ with an active volume of 65 liter. It was basically designed for ${ }^{222} \mathrm{Rn}$ concentration measurements in nitrogen, but can also be used for ${ }^{222} \mathrm{Rn}$ monitoring in dry air or emanation tests. The minimum detectable activity (MDA) for the present system is $0.8 \mathrm{mBq} \quad\left(12 \mathrm{mBq} / \mathrm{m}^{3}\right)$ assuming $30 \%$ accuracy. Based on the experience gained with the prototype, a detector with the volume of $1 \mathrm{~m}^{3}$ is under consideration. The increase in volume, combined with anticipated background reduction, should provide a sensitivity level of $0.5 \mathrm{mBq} / \mathrm{m}^{3}$ or less.

\section{Experimental}

\section{Detector design}

The cross section of the cryogenic detector is shown in Fig. 1. It consists of three main parts: a special Dewar for cryogenic liquid (cryo-reservoir) with an integrated electropolished stainless steel plate (cold plate, collector), a detector chamber, and the large Ortec silicon detector for alpha-spectrometry. The system is made of stainless steel with all inner surfaces electropolished and carefully cleaned in order to minimize background originating from ${ }^{222} \mathrm{Rn}$ emanation.

The cryogenic liquid reservoir is a double-wall vacuum-insulated container. It is equipped with a flange to mount it in the detector chamber, a valve for a vacuum pump connection, and a BNC signal feed-through. The flange is sealed with two O-rings: polyurethane (outer gasket) and indium (inner gasket). This combination assures tightness of the system and minimizes ${ }^{222} \mathrm{Rn}$ diffusion from outside into the active volume.

The cold plate is integrated with the cryo-reservoir as shown in Fig. 1. The diameter of the area directly cooled with $\mathrm{LN}_{2}$ is $d=40 \mathrm{~mm}$ and its thickness is $0.5 \mathrm{~mm}$. Thanks to this design the temperature of the collector on the detector-chamber side is very close to that of the boiling point of the liquid used for cooling.

The diameter of the active surface of the Ortec diode is $D=50 \mathrm{~mm}$. It is centered on, and facing the cold plate. The semiconductor detector is a standard Ortec alphaspectroscopy design. The distance $H$ between the collector and the diode surface can be manually varied between 1 and $30 \mathrm{~mm}$. All tests and measurements described below were carried out for $H=12 \mathrm{~mm}$. 
It was chosen as an optimum taking into account the counting efficiency and the desirable working conditions of the semiconductor.

\section{Background, efficiency, and the ${ }^{222}$ Rn minimum detectable activity of the detector}

The background of the cryogenic detector has two independent components. The first component is generated by a combination of alpha-emitting impurities in the semiconductor diode (especially in the active surface layer), and cosmic ray events. Its measurement was carried out with the chamber evacuated, and no cryogen in the reservoir (to avoid adsorbing alphaemitters on the cold plate). The background signal in the ${ }^{222} \mathrm{Rn}$ region of interest is $A_{B 0}=(0.93 \pm 0.31)$ counts per day (cpd).

The second component is related to the permanent production of ${ }^{222} \mathrm{Rn}$ inside the chamber (emanation from the welds, valves or others components). This background contribution becomes apparent after filling the evacuated cryo-reservoir with liquid nitrogen, when traces of ${ }^{222} \mathrm{Rn}$ present in the 65 liter chamber are collected on the cold plate. The total background was measured with the same procedure used for sample investigations, and is as follows: (1) some hours before the measurement, fill (with $\mathrm{N}_{2}$ ) and evacuate the chamber several times; (2) immediately prior to filling with ${ }^{222} \mathrm{Rn}$, evacuate the chamber down to $10^{-2} \mathrm{mbar}$ and close; (3) one hour later fill the cryo-reservoir with $\mathrm{LN}_{2}$. This time delay corresponds to the period needed to introduce a sample into the detector. During that hour outgassing ${ }^{222} \mathrm{Rn}$ accumulates inside the chamber and is later collected on the plate; and (4) start the measurement 30 minutes after filling the cryo-reservoir (at low pressure this time is sufficient to freeze almost all ${ }^{222} \mathrm{Rn}$ on the collector - see next chapters). Record the count rates of alpha-decays in the energy windows of ${ }^{222} \mathrm{Rn},{ }^{218} \mathrm{Po}$ and ${ }^{214} \mathrm{Po}$.

The background measurement was performed three times, with an average of: $A_{B}=24.5 \pm 3.5 \mathrm{cpd}$. It is clear that the signal from ${ }^{222} \mathrm{Rn}$ emanating inside the detector chamber is very strong $(23.6 \mathrm{cpd})$ compared to the Ortec diode background of $0.9 \mathrm{cpd}$.

The absolute detection efficiency $\varepsilon$ for the low gas (nitrogen or helium) pressure in the detector ( $\sim 2$ mbar) was determined in the following way:

A bubbler of the volume of $20 \mathrm{~cm}^{3}$ containing a water solution with well known ${ }^{226} \mathrm{Ra}$ activity (a standard) was flushed with gaseous nitrogen (class 5.0) two weeks before the measurement. This purged all ${ }^{222} \mathrm{Rn}$ from the source and allowed precise calculation of the ${ }^{222} \mathrm{Rn}$ activity at any later time.

The detector was connected with the bubbler through a humidity (water) trap.
The trap and the detector chamber were evacuated.

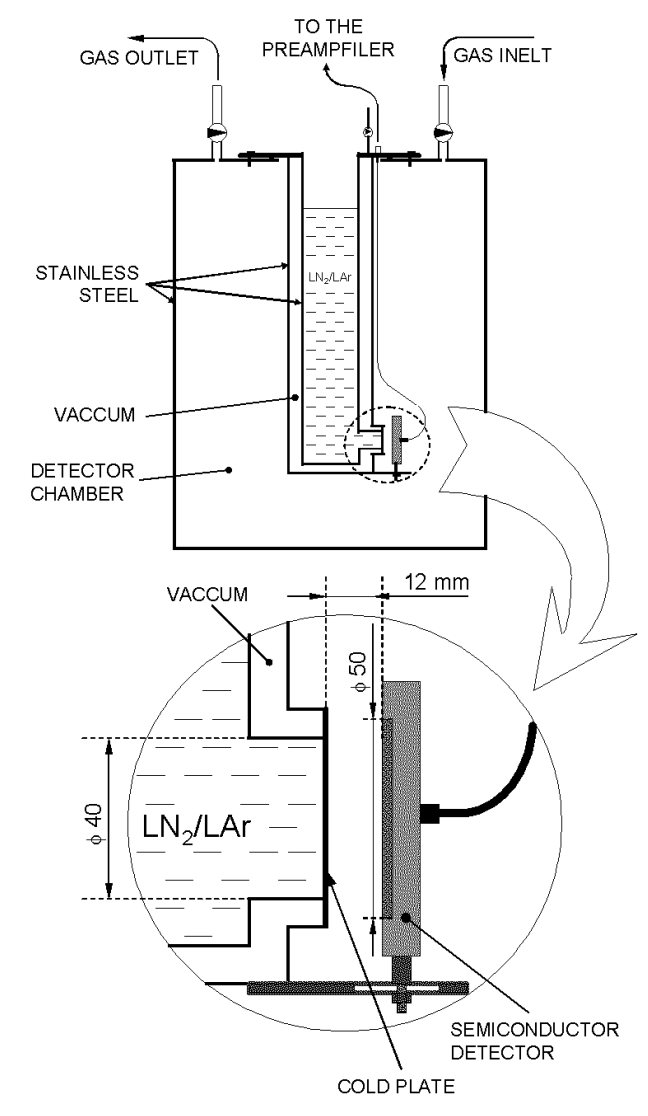

Fig. 1. Scheme of the cryogenic detector for radon

The humidity trap was cooled to $-72{ }^{\circ} \mathrm{C}$ using ethanol. About $100 \mathrm{~cm}^{3}$ (STP) of nitrogen was flushed through the bubbler and the trap into the detector. This amount of gas was sufficient to extract all ${ }^{222} \mathrm{Rn}$ from the solution.

The detector reservoir was filled with liquid nitrogen.

After 3 hours, when all radon was collected on the cold plate and the radioactive equilibrium between ${ }^{222} \mathrm{Rn}$ and its short lived daughters has been established, the data acquisition was started.

The average absolute detection efficiency (from three independent measurements) for nitrogen carrier gas at the pressure of about 2 mbar is $\varepsilon_{N}=(31.2 \pm 0.9) \%$. A similar test with helium (class 5.0) carrier provided a measured efficiency equivalent to that above (within error) $\varepsilon_{H e}=(31.7 \pm 0.9) \%$. Finally one can conclude that for low pressure in the detector chamber and for cooling using $\mathrm{LN}_{2}$ :

$$
\varepsilon=\left(\varepsilon_{N}+\varepsilon_{H e}\right) / 2=(31.5 \pm 0.6) \%
$$

From the registered energy spectrum one can also extract the energy resolution (FWHM) for alphaparticles emitted by ${ }^{222} \mathrm{Rn}$, which is $\sim 105 \mathrm{keV}$ (at low gas pressure). 
On the basis of the measured background $A_{B}$ and the detection efficiency $\varepsilon$ we have found the minimum detectable activity of ${ }^{222} \mathrm{Rn}^{5}$ for the prototype detector. In the calculations we took into account only radon (without its daughters). The minimum detectable activity can be found according the following formula:

$$
\delta=\frac{\sigma_{A_{0}(0)}}{A_{0}(0)}
$$

where $A_{0}(0)$ is the activity for $t=0$ (the time when the detector is filled), $\sigma_{A_{0}(0)}$ is its standard deviation, and $\delta$ represents the relative error of the measurement, which is assumed a priori. $A_{0}(0)$ and $\sigma_{A_{0}(0)}$ can be found from the equation describing the total number of counts $N$ registered in the time interval $\Delta t$ :

$$
N=\varepsilon \int_{t_{s}}^{t_{s}+\Delta t} A_{0}(t) \mathrm{d} t+A_{B} \Delta t
$$

where $A_{0}(t)$ describes the ${ }^{222} \mathrm{Rn}$ activity for the time $t$, and $t_{s}$ denotes the time between the ${ }^{22} \mathrm{Rn}$ introduction into the detector and the measurement start. Calculation of $\sigma_{A_{0}}(0)$ takes into account the uncertainties of the detector background $\left(\sigma_{A_{B}}\right)$ and the efficiency $\left(\sigma_{\varepsilon}\right)$. Finally we get:

$$
=\frac{\lambda e^{\lambda t_{s}\left(1+\sqrt{1+4\left(\Delta t^{2} \sigma_{A_{B}}^{2}+\Delta t A_{B}\right)\left(\delta^{2}-\delta_{\varepsilon}^{2}\right)}\right)}}{2 \varepsilon\left(1-e^{-\lambda \Delta t}\right)\left(\delta^{2}-\delta_{\varepsilon}^{2}\right)}
$$

where $\lambda$ is the ${ }^{222} \mathrm{Rn}$ decay constant and $\delta_{\varepsilon}=\left(\sigma_{\varepsilon} / \varepsilon\right)$ is the relative error of the absolute detection efficiency.

Taking into account previously determined parameters of the cryogenic detector and assuming $t_{s}=1.5$ hours one can calculate $A_{0}(0)_{\min }$ for different $\delta$ values. Results are collected in Table 1 , where $\Delta t_{\text {min }}$ is the measurement time corresponding to the calculated $A_{0}(0)_{\min }$ and $A_{0}(0)_{1000}$ denotes the activity obtained for the assumed measurement time of $1000 \mathrm{~min}$. The plot in the Fig. 2 shows $A_{0}(0)_{\min }$ as a time function for $\delta=0.3$.

As can be seen, assuming a relative accuracy of $30 \%$, the MDA for the prototype detector is about $0.8 \mathrm{mBq}$. Taking into account the active volume of the chamber $(651)$ one gets the minimum detectable concentration of ${ }^{222} \mathrm{Rn}$ at the level of $12 \mathrm{mBq} / \mathrm{m}^{3}$. For a 1000-minute measurement the activity and concentration can be measured with $30 \%$ uncertainty at $1.3 \mathrm{mBq}$ and $21 \mathrm{mBq} / \mathrm{m}^{3}$, respectively.

The sensitivity of the detector can be improved by reducing the background and increasing the overall detection efficiency. Hypothetically reducing the background by half (together with its error) down to $(12.8 \pm 1.7) \mathrm{cpd}$ one could get $A_{0}(0)_{\min }=0.57 \mathrm{mBq}$ for a measurement time of 6644 minutes. Decreasing the distance between the Ortec diode and the collector to $3 \mathrm{~mm}$ should increase the total detection efficiency up to $45 \%$. As a result one could further reduce the MDA to the level of $0.36 \mathrm{mBq}$. Simultaneous increase of the detector volume up to $1 \mathrm{~m}^{3}$ would allow us to measure ${ }^{222} \mathrm{Rn}$ concentrations down to $0.36 \mathrm{mBq} / \mathrm{m}^{3}$. However, one should consider a possible degradation of the ${ }^{222} \mathrm{Rn}$ collection efficiency on the cold plate, especially for the higher gas pressure - see next chapters.

\section{Influence of the carrier gas type and pressure on the ${ }^{222}$ Rn collection on the cold plate}

The aim of the following measurements was to determine the time needed to quantitatively collect radon from the gas present in the detector volume (at different pressures) on the cold plate. Knowledge of this parameter is necessary to characterize the usefulness of the device to applications where the measurement cycle time is limited. The cryogenic detector was tested using $\mathrm{LN}_{2}$ as a cooling liquid and nitrogen and helium as carrier gases. The measurement procedure was identical for both and is described below.

Prepare of a proper ${ }^{222} \mathrm{Rn}$ source (activity of some $\mathrm{kBq}$ ) two weeks before each test. Flush the source chamber with nitrogen or helium in order to remove accumulated ${ }^{222} \mathrm{Rn}$.

Connect the source with the detector through a humidity (water) trap using vacuum tubes.

Evacuate the detector chamber and humidity trap.

Close the valve between the water trap and the detector.

Cool the humidity trap to $-72{ }^{\circ} \mathrm{C}$ using cold ethanol.

Slowly open the valve connecting the ${ }^{222} \mathrm{Rn}$ source and the water trap. Close the valve after the gas $\left(\mathrm{N}_{2}+{ }^{222} \mathrm{Rn}\right.$ or $\left.\mathrm{He}^{+222} \mathrm{Rn}\right)$ fills the moisture trap.

Open the valve connecting the trap with the detector. The dry gas moves into the detector chamber through volumetric expansion. (The gas pressure in the detector chamber after filling it with $\mathrm{Rn}$ (together with a few $\mathrm{cm}^{3}$ of $\mathrm{N}_{2} / \mathrm{He}$ ) was very low and on all plots and in tables is denoted as "0".)

Table $1 .{ }^{222} \mathrm{Rn}$ minimum detectable activity calculated for different measurement precisions

\begin{tabular}{lccc}
\hline \multicolumn{1}{c}{$\delta$} & $A_{0}(0)_{\min }, \mathrm{mBq}$ & $\Delta t_{\min }, \min$ & $A_{0}(0)_{1000}, \mathrm{mBq}$ \\
\hline 0.05 & 8.2 & 9126 & 27.8 \\
0.1 & 3.0 & 6752 & 7.0 \\
0.15 & 1.8 & 5874 & 3.5 \\
0.2 & 1.3 & 5402 & 2.3 \\
0.25 & 1.0 & 5105 & 1.6 \\
0.3 & 0.82 & 4900 & 1.3 \\
0.4 & 0.59 & 4635 & 0.88 \\
0.5 & 0.47 & 4470 & 0.67 \\
0.6 & 0.38 & 4358 & 0.54 \\
\hline
\end{tabular}




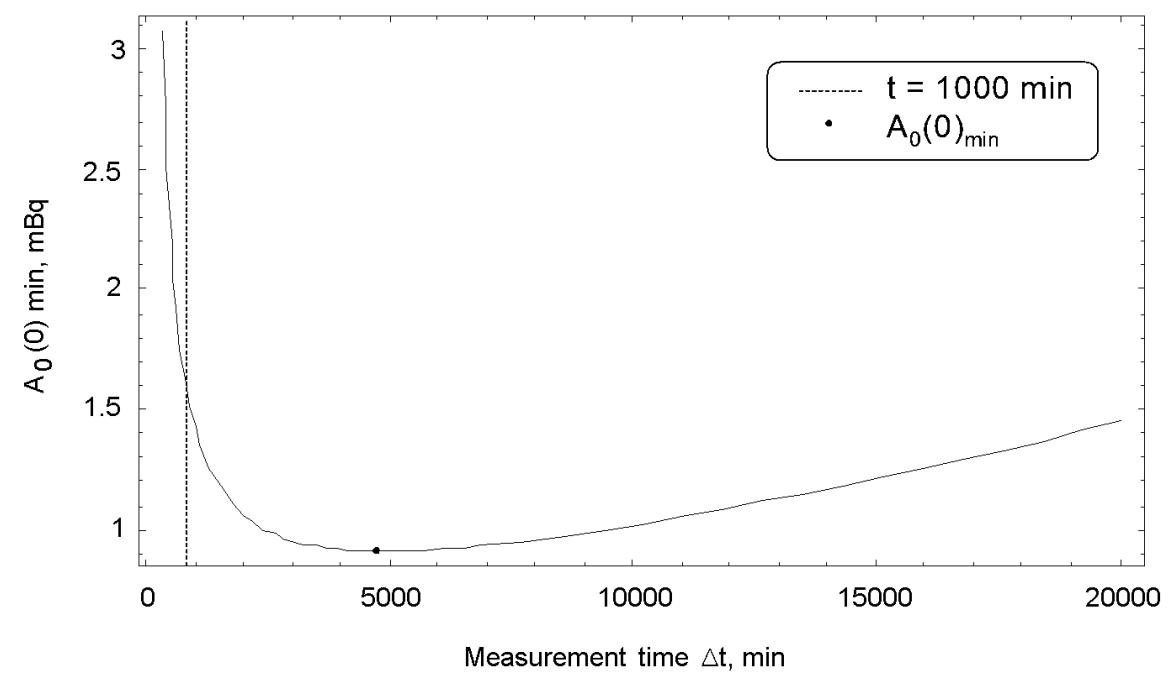

Fig. 2. Dependence of the ${ }^{222} \mathrm{Rn}$ minimum detectable activity on the measurement time for the obtained detector parameters $\left(A_{B}\right.$ and $\left.\varepsilon\right)$ and for the relative measurement accuracy of $30 \%$

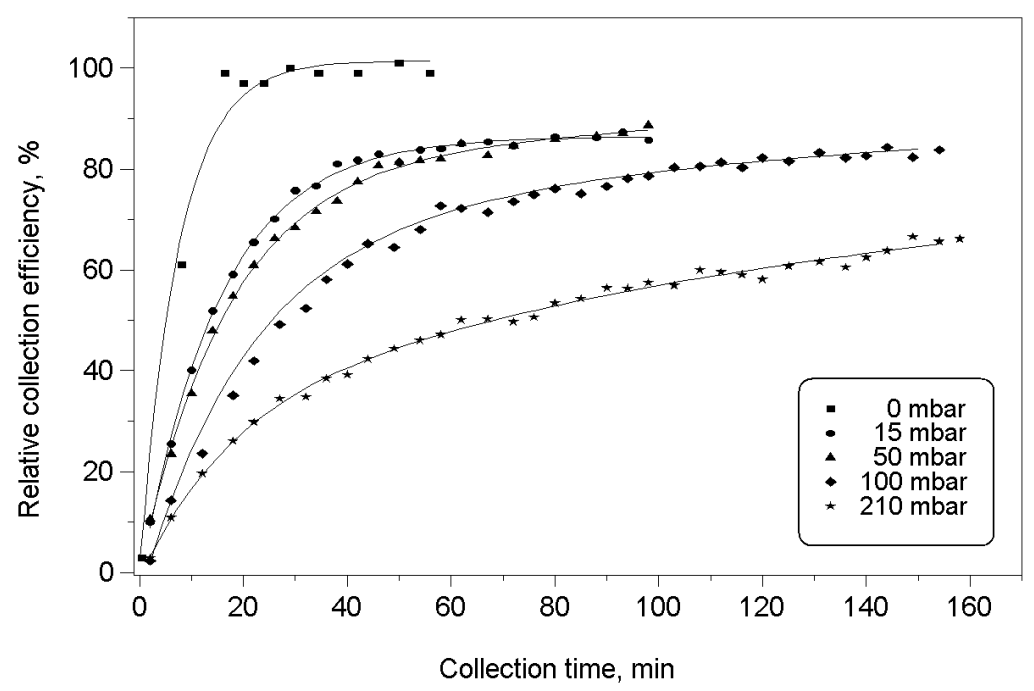

Fig. 3. Dependence of the ${ }^{222} \mathrm{Rn}$ relative detection efficiency $\left({ }^{222} \mathrm{Rn}\right.$ collection speed) on the time and nitrogen pressure in the detector volume

Fill the detector reservoir with $\mathrm{LN}_{2}$; collection of ${ }^{222} \mathrm{Rn}$ on the cold plate starts.

Register in time periods (some minutes) the number of counts in the energy windows corresponding to alphaparticles emitted by ${ }^{222} \mathrm{Rn},{ }^{218} \mathrm{Po}$ and ${ }^{214} \mathrm{Po}$.

In most cases while investigating the dependence of the collecting efficiency on the gas (nitrogen or helium) pressure, before introducing it into the chamber the $\mathrm{LN}_{2}$ reservoir was emptied what resulted in a ${ }^{222} \mathrm{Rn}$ desorption from the plate.

Fill the detector chamber with $\mathrm{N}_{2}$ or He from a gas cylinder to the desired pressure $p$.
Fill the reservoir with $\mathrm{LN}_{2}$ and start the observation of ${ }^{222} \mathrm{Rn}$ collection speed for the pressure $p$.

The ${ }^{222} \mathrm{Rn}$ collection on the plate cooled by $\mathrm{LN}_{2}$ was investigated for the following nitrogen pressures: $\sim 0$ mbar, 15 mbar, 50 mbar, 100 mbar, 210 mbar and for helium pressures $\sim 0$ mbar, 130 mbar and 400 mbar. For each case during the measurement the relative detection efficiency $\varepsilon_{R e l}$ was determined. Its highest value was reached for the lowest pressure $\left(p \sim 2\right.$ mbar for $\mathrm{N}_{2}$ and $\mathrm{He})$ and was taken as $\varepsilon_{R e l}=100 \%$. Figure 3 shows the dependence of the ${ }^{222} \mathrm{Rn}$ collection speed on the time for different pressures of nitrogen. In Fig. 4 similar dependence for He is shown. 


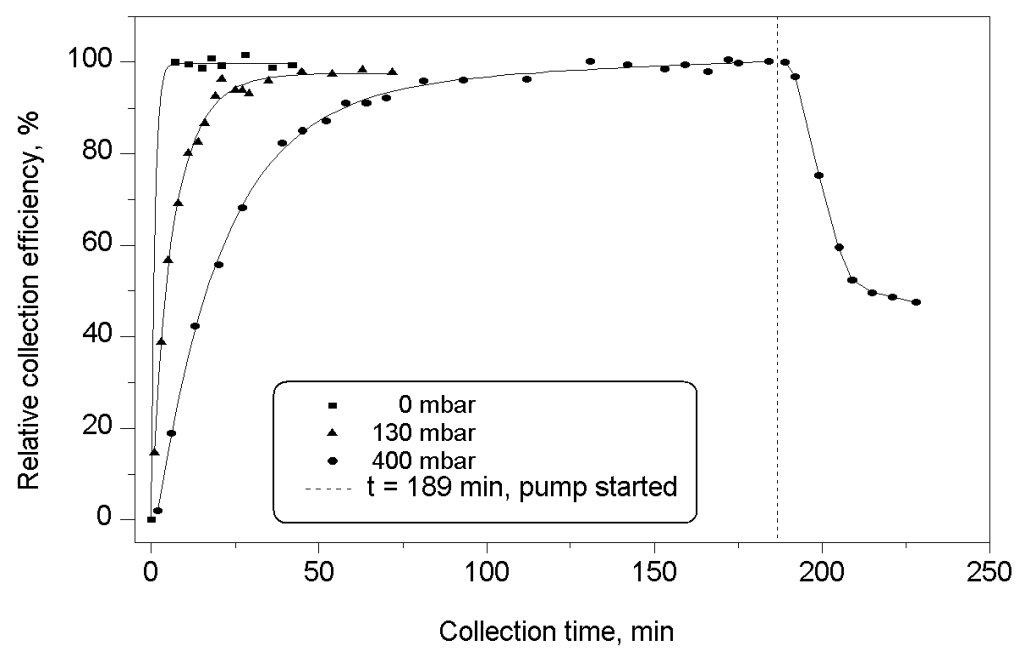

Fig. 4. Dependence of the ${ }^{222} \mathrm{Rn}$ relative detection efficiency on the He pressure in the detector chamber. For the pressure of $400 \mathrm{mbar}$ after the collection time of 189 minutes the detector chamber was pumped down. As a result the counting rate dropped down by $50 \%$

The absolute detection efficiency was measured for the pressure of $p \sim 2$ mbar using a standard water solution of ${ }^{226} \mathrm{Ra}$ of the activity of $0.802 \mathrm{~Bq}$. It was assumed that the efficiency will be the same for higher ${ }^{222} \mathrm{Rn}$ concentrations and a similar pressure. This means that for the relative efficiency of $\varepsilon_{R e l}=100 \%$ but higher ${ }^{222} \mathrm{Rn}$ activity the absolute detection efficiency is $(31.5 \pm 0.6) \%$. For higher pressures the absolute detection efficiency was determined using measured earlier values of relative efficiencies. Table 2 gathers all the results of absolute efficiencies as a function of nitrogen pressure in the detector chamber. Table 3 shows similar results obtained for different He pressures.

Table 2. Absolute registration efficiency of ${ }^{222} \mathrm{Rn}$ and the measured count rate ratios of ${ }^{218} \mathrm{Po} /{ }^{222} \mathrm{Rn}$ for five different nitrogen pressures

\begin{tabular}{ccc}
\hline $\begin{array}{c}\text { Pressure } \\
(p), \text { mbar }\end{array}$ & $\begin{array}{c}\text { Absolute efficiency } \\
(\varepsilon), \%\end{array}$ & Ratio $^{218} \mathrm{Po} /{ }^{22} \mathrm{Rn}$ \\
\hline$\sim 0$ & $31.5 \pm 0.6$ & $1.088 \pm 0.016$ \\
15 & $27.2 \pm 0.7$ & $0.966 \pm 0.014$ \\
50 & $28.1 \pm 0.7$ & $0.945 \pm 0.013$ \\
100 & $26.2 \pm 0.9$ & $0.965 \pm 0.014$ \\
210 & $20.7 \pm 1.1$ & $0.849 \pm 0.013$ \\
\hline
\end{tabular}

Table 3. Absolute registration efficiency of ${ }^{222} \mathrm{Rn}$ and the count rate ratios of ${ }^{218} \mathrm{Po} /{ }^{222} \mathrm{Rn}$ at steady state conditions for nine different helium pressures

\begin{tabular}{ccc}
\hline $\begin{array}{c}\text { Pressure } \\
(p), \text { mbar }\end{array}$ & $\begin{array}{c}\text { Absolute efficiency } \\
(\varepsilon), \%\end{array}$ & Ratio $^{218} \mathrm{Po} / 222 \mathrm{Rn}$ \\
\hline 0 & $31.5 \pm 0.6$ & $1.064 \pm 0.011$ \\
20 & $31.6 \pm 0.6$ & $1.064 \pm 0.011$ \\
40 & $31.5 \pm 0.6$ & $1.037 \pm 0.011$ \\
50 & $31.4 \pm 0.6$ & $1.043 \pm 0.011$ \\
130 & $31.5 \pm 0.6$ & $1.019 \pm 0.011$ \\
200 & $31.5 \pm 0.6$ & $1.011 \pm 0.014$ \\
305 & $31.3 \pm 0.6$ & $1.002 \pm 0.014$ \\
370 & $30.1 \pm 0.7$ & $0.987 \pm 0.012$ \\
400 & $31.5 \pm 0.6$ & $0.988 \pm 0.012$ \\
\hline
\end{tabular}

After ${ }^{222} \mathrm{Rn}$ was collected on the cold plate and when the equilibrium between its adsorption and desorption is reached one can expect that a radioactive equilibrium between ${ }^{222} \mathrm{Rn}$ and its daughters will be established. After the first 15 minutes the activities of ${ }^{218} \mathrm{Po}$ and ${ }^{222} \mathrm{Rn}$ are equal. ${ }^{214} \mathrm{Po}$ is in equilibrium with ${ }^{222} \mathrm{Rn}$ after about 3 hours. In Tables 2 and 3 the third columns contain the calculated ratios of ${ }^{218} \mathrm{Po}$ and ${ }^{222} \mathrm{Rn}$ count rates.

\section{Results and discussion}

The background is one of the most important parameters of the detector which determines its sensitivity. The prototype detector presented here used a standard (not an ultra low background) Ortec semiconductor diode that registers less than $1 \mathrm{cpd}$ for an energy window corresponding to ${ }^{222} \mathrm{Rn}$ alpha-decays. This rate does not significantly influence the ${ }^{222} \mathrm{Rn}$ minimum detectable activity of the cryogenic detector. Its main source of background comes from radon emanation from internal welds, gaskets, valves and surfaces of the chamber (about $\sim 0.8 \mathrm{~m}^{2}$ of electropolished stainless steel). Investigations carried out in the frame of the Borexino experiment ${ }^{6}$ show that the ${ }^{222} \mathrm{Rn}$ emanation from a clean steel surface is very low $\left(\sim 5 \mu \mathrm{Bq} / \mathrm{m}^{2}\right)$ and in many cases welds may be a main source of ${ }^{222} \mathrm{Rn}$ (e.g., when using rods containing thorium and uranium/radium). Our experience also points that the careful selection of materials used to construct the detector (gaskets, valves) can reduce its background far below the $24 \mathrm{cpd}$ found in the prototype. 
Another important parameter of a low-level detector is its absolute detection efficiency for a given type of radiation. In our case it can be written as $\varepsilon=\varepsilon_{c} \varepsilon_{g} \varepsilon_{d}$, where $\varepsilon_{c}$ is the efficiency of ${ }^{222} \mathrm{Rn}$ collection on the cold plate, $\varepsilon_{g}$ depends on the diode - collector geometry and $\varepsilon_{d}$ denotes the detection probability of an alpha particle emitted towards the diode. For the geometry presented $(D=50 \mathrm{~mm}, d=40 \mathrm{~mm}, H=12 \mathrm{~mm})$ one can calculate $\varepsilon_{g}$, which is about $34 \%$. Measured absolute detection efficiency is $\varepsilon=31.5 \%$. Taking into account the detector design it is possible that $\varepsilon_{g}$ is in reality lower that the calculated value. The reason is that the ${ }^{222} \mathrm{Rn}$ can be collected on the surface with the diameter higher than $d$. Liquid nitrogen cools directly the area of the $40 \mathrm{~mm}$ diameter but due to the steel heat conductivity ${ }^{222} \mathrm{Rn}$ can also be frozen a few $\mathrm{mm}$ outside of that area. Also $\varepsilon_{c}$ and $\varepsilon_{d}$ are lower than 1 and dependent on the type and pressure of the gas ${ }^{222} \mathrm{Rn}$ is collected from. It is very difficult to freeze ${ }^{222} \mathrm{Rn}$ from nitrogen of high pressure ( $\varepsilon_{c}$ is significantly lower compared to vacuum). For example, for scintillation chambers the value of $\varepsilon_{d}$ drops exponentially with increasing gas pressure. ${ }^{7}$ This effect is related to the increase of disperse centers on the alphas way from the source to the detector. In our case an additional effect of $\varepsilon_{d}$ degradation may appear, namely condensation of the carrier gas on the cold plate. This affects, e.g., measurements of ${ }^{222} \mathrm{Rn}$ in nitrogen when $\mathrm{LN}_{2}$ is used as a cooling medium.

This effect may be also responsible for the change of positions and width of the energy windows in which alpha-particles emitted by ${ }^{222} \mathrm{Rn}$ and its daughter are registered. For higher gas pressures the alpha-peaks broaden and move towards lower energies. For the discussed geometry and nitrogen pressure of $210 \mathrm{mbar}$ the ${ }^{222} \mathrm{Rn}$ peak is so broad that further pressure increase results in overlap of the ${ }^{222} \mathrm{Rn}$ and ${ }^{218}$ Po peaks. On the other hand collecting ${ }^{222} \mathrm{Rn}$ from He at 400 mbar takes about 150 minutes (after that time equilibrium between ${ }^{222} \mathrm{Rn}$ adsorption and desorption is established) and the peaks are still narrow enough to avoid their overlap. This result supports our assumption about gas condensation on the collector; it appears that thin layer of, e.g., nitrogen may form on the steel surface. This degrades both ${ }^{222} \mathrm{Rn}$ collection and detection of alphas. This problem can be solved by using liquid argon for cooling when ${ }^{222} \mathrm{Rn}$ in nitrogen is measured (in general the boiling point of cooling medium should be higher than that for the carrier gas).

Tests showed that the collection efficiency of ${ }^{222} \mathrm{Rn}$ from large volumes depends strongly on the carrier gas type and pressure. For example ${ }^{222} \mathrm{Rn}$ equilibrium between ${ }^{222} \mathrm{Rn}$ adsorption and desorption from nitrogen at 100 mbar is reached after 120 minutes, but when using helium at 130 mbar the ${ }^{222} \mathrm{Rn}$ collection is completed after 45 minutes. In both cases $\mathrm{LN}_{2}$ was used for cooling. In general, for higher pressures the ${ }^{222} \mathrm{Rn}$ collection time on the cold plate is longer and the overall detection efficiency is lower.

Attempts to remove the carrier gas $\left(\mathrm{He}\right.$ or $\left.\mathrm{N}_{2}\right)$ from the active volume of the chamber after ${ }^{222} \mathrm{Rn}$ was frozen on the plate always led to simultaneous removal of ${ }^{222} \mathrm{Rn}$ (Fig. 4). ${ }^{222} \mathrm{Rn}$ atoms are desorbed and pumped out. For He at 400 mbar 26 minutes of pumping resulted in a $50 \%$ reduction of the count rate below the saturation value. In the case of nitrogen pumping from $210 \mathrm{mbar}$ reduced the counting rate by $25 \%$ after 19 minutes. Six measurements of the absolute counting efficiency were performed using a standard ${ }^{222} \mathrm{Rn}$ source of the activity of $0.802 \mathrm{~Bq}$. In all cases ${ }^{222} \mathrm{Rn}$ was introduced into the detector chamber with a small amount of carrier gas (end pressure $\sim 2$ mbar) and after ${ }^{222} \mathrm{Rn}$ was collected on the plate the chamber evacuation was started. For all tests the efficiencies were very low, in the order of 10 $12 \%$.

The effect observed shows that not all ${ }^{222} \mathrm{Rn}$ was adsorbed on the steel surface $\left(\varepsilon_{c}<1\right)$. In the detector volume a dynamic equilibrium between ${ }^{222} \mathrm{Rn}$ deposition and desorption is established. For higher pressures more ${ }^{222} \mathrm{Rn}$ stays in the gas phase, decreasing the detection efficiency. For radioactive equilibrium between ${ }^{222} \mathrm{Rn}$ and ${ }^{218} \mathrm{Po}$ the ratio $\left(A_{\mathrm{Po} 218} / A_{\mathrm{Rn} 222}\right)$ should be higher than 1 . This is observed only for low pressure in the detector chamber. For higher $p$ values that factor is clearly lower than 1 . This effect could be explained by a presence of high number of atoms between the source (collector) and the diode. These atoms can reduce the probability that ${ }^{218} \mathrm{Po}$ will return to the cold plate after it was ejected due to the ${ }^{222} \mathrm{Rn}$ decay (after ${ }^{222} \mathrm{Rn}$ decay ${ }^{218} \mathrm{Po}$ is created in most cases as a positive ion and gets a recoil energy in the range of $100 \mathrm{keV})$.

\section{Conclusions}

As mentioned, the detector was built in principle as a prototype and was used to check the performance of such a device. A new system under consideration would have the chamber volume on the order of $1 \mathrm{~m}^{3}$ with simultaneous background reduction. The target sensitivity would be $1 \mathrm{mBq} / \mathrm{m}^{3}$ or less.

Another possibility is to build a detector with a rather small volume, with a vacuum flange allowing it to be connected to a separate chamber. Such a design could also be used to monitor ${ }^{222} \mathrm{Rn}$ content in a gas stream (online monitoring). The good energy resolution of the ORTEC detector also provides the possibility to measure ${ }^{220} \mathrm{Rn}$ and its daughters simultaneously with ${ }^{222} \mathrm{Rn}$.

$*$

This work was partially supported by the Polish Scientific Research Committee, Grant No. 2 PO3B 15522. 


\section{References}

1. W. RaU, G. HeUSSER, Appl. Radiation Isotopes, 53 (2000) 371

2. R. WINK et al., Nucl. Instr. Meth., A329 (1993) 541.

3. J. KIKO, Nucl. Instr. Meth., A460 (2001) 272.

4. Y. TAKEUCHI et al., Nucl. Instr. Meth., A421 (1999) 334.

5. M. WoJcIK, W. Wlazlo, Nucl. Instr. Meth., A345 (1994) 351.
6. Radon Emanation Measurements for the Borexino Experiment, Borexino Internal Report, 2002.

7. M. WoJcIK, Environmental ${ }^{222} \mathrm{Rn}$ as a Background Source in the Solar Neutrino Experiment GALLEX, Report No. 1729/PH, Institute of Nuclear Physics, Cracow, Poland, 1997. 